\title{
Clinical Medicine Insights: Circulatory, Respiratory and Pulmonary Medicine
}

\section{Correlation of Stroke Volume Measurement between Sonosite Portable Echocardiogram and Edwards Flotrac Sensor-Vigileo Monitor in an Intensive Care Unit}

\author{
Mehrdad Behnia ${ }^{1-3}$, Sherry Powell ${ }^{2}$, Linda Fallen², Houman Tamaddon ${ }^{3}$ and Masud Behnia ${ }^{3}$ \\ ${ }^{1}$ Georgia Health Sciences University, ${ }^{2}$ Doctors Hospital, Augusta, GA. ${ }^{3}$ School of Mechanical and Mechatronic \\ Engineering, The University of Sydney, Sydney, Australia. Corresponding author email: doctorbehnia@gmail.com
}

\begin{abstract}
Purpose: Stroke volume (SV) is a parameter that is being recognized as an endpoint in fluid resuscitation algorithms. Its role is now being realized as an important variable in hemodynamic assessment in various clinical scenarios such as septic and cardiogenic shocks. Direct measurement of stroke volume (SV) and its novel corollary, stroke volume variation (SVV) derived by proprietary software, are preferred over mean cardiac output $(\mathrm{CO})$ measurements because they render a more accurate reflection of hemodynamic status independent of heart rate. Flotrac-Vigileo monitor (FTV) (Edwards Lifesciences, Irvine, CA, USA) is a system that uses a complex algorithm analyzing arterial waveform to calculate SV, SVV, and CO. We assessed the feasibility of obtaining SV measurements with a portable echocardiogram and validated its accuracy with the FTV system in mechanically ventilated patients in our intensive care unit (ICU). Furthermore, we emphasized the importance of hemodynamic measurements and familiarity with critical care echocardiography for the intensivists.

Methods: Ten patients who were on mechanical ventilation were studied. A femoral arterial line was connected to the FTV system monitoring SV and CO. A portable echocardiogram (M-Turbo; Sonosite, Bothell, WA) was used to measure SV. CO was calculated by multiplying SV by heart rate. No patient had arrhythmia. We used biplane Simpson's method of discs to calculate SV in which subtraction of end-systolic volume from end-diastolic volume yields the SV.

Results: The comparison of simultaneous SV and CO measurements by echocardiography with FTV showed a strong correlation between the 2. (For SV, $\mathrm{y}=0.9545 \mathrm{x}+3.3, R^{2}=0.98$ and for $\mathrm{CO}, \mathrm{y}=0.9104 \mathrm{x}+7.7074, R^{2}=0.97$ ).

Conclusions: In our small cohort, the SV and CO measured by a portable echocardiogram (Sonosite M-Turbo) appears to be closely correlated with their respective values measured by FTV. Portable echocardiography is a reliable noninvasive tool for the hemodynamic assessment of the critically ill. Its results need further validation with gold standard measures in a larger cohort of patients. However, our results suggest portable echocardiography could be an attractive tool in assessment of different hemodynamic scenarios in the critically ill.
\end{abstract}

Keywords: noninvasive hemodynamics, cardiac output, monitoring, stroke volume variation

Clinical Medicine Insights: Circulatory, Respiratory and Pulmonary Medicine 2013:7 45-51

doi: $10.4137 / C C R P M . S 12498$

This article is available from http://www.la-press.com.

(c) the author(s), publisher and licensee Libertas Academica Ltd.

This is an open access article published under the Creative Commons CC-BY-NC 3.0 license. 


\section{Introduction}

Hemodynamic monitoring and fluid resuscitation is of paramount importance in any intensive care unit (ICU). The challenging question for a clinician is what method and what endpoint must be used to assess the hemodynamic status of a patient? This has yet to be agreed on uniformly by all clinicians taking care of the critically ill. An ideal parameter must have a high sensitivity and be reproducible in most clinical scenarios. Another very important factor is the invasive or noninvasiveness nature of a parameter. ${ }^{1}$ Pulmonary artery catheterization was once an ideal tool in hemodynamic monitoring; however, throughout the years it lost its popularity and is not being used as frequently as it was used previously. ${ }^{1}$

Another device that was introduced recently into the arena of hemodynamic monitoring is the Flotrac-Vigileo monitor (FTV) (Edwards Lifesciences, Irvine, CA, USA). This system requires placement of an arterial line and uses complex software based on pressure waveform analysis for measuring $\mathrm{CO}$ and $\mathrm{SVV}^{2}$ The system has gained popularity among researchers and clinicians as a tool that is associated with lesser complications and is technically easier to insert and operate compared with the pulmonary artery catheter. Its limitations are that (1) it requires placement of an arterial line and (2) it is mainly reliable in mechanically ventilated patients.

An underutilized alternative method in hemodynamic assessment of the critically ill is portable echocardiography, which can be reliable in both ventilated and nonventilated patients. Bedside echocardiography has long been accepted as a reliable tool in hemodynamic monitoring, preload assessment, and volume appraisal by clinicians. Its noninvasive nature makes it an ideal tool that can be used in a wide variety of clinical scenarios. Numerous citations have attested to its clinical efficacy. ${ }^{3}$

However, portable echocardiography has not been well accepted by a group of clinicians who consider invasive measurements such as pulmonary artery catheterization and other tools such as FTV more reliable tools in hemodynamic monitoring in the ICU.

Portable echocardiography is not only noninvasive but can also be used readily in places that invasive hemodynamic monitoring is unattainable. Examples include small nontertiary hospitals, combat field medical settings, and smaller hospitals in countries where invasive hemodynamic monitoring is still not widely accepted or available. Sonosite(Bothell, WA, USA) portable (M-Turbo) echocardiogram makes it an ideal system that can be readily available in these settings.

In the present study, we sought to compare the practicality, ease of use, and accuracy of the Sonosite M-Turbo echocardiogram relative to the advanced invasive hemodynamic monitoring of the FTV system. Stroke volume and cardiac output measurement by echocardiography and FTV system were compared. Patient characteristics and other hemodynamic variables were also measured and compared between the 2 methods. We hypothesized that in the critical care setting, the noninvasive assessment of SV using the portable echocardiography system would closely track measures obtained from the invasive FTV system.

\section{Methods}

The study protocol was reviewed and approved by the ethics committee and institutional committee boards. Ten critically ill patients who were all intubated and were on positive pressure mechanical ventilation and were receiving continuous intravenous sedation were studied. All had a 20 guage arterial line (Arrow International/ARROW International-Teleflex; Cleveland, Ohio, USA) placed as a routine requirement for patients on mechanical ventilation. Their characteristics are elaborated in Table 1.

The FTV was set up based on the manufacturing guidelines of the manufacturer by connecting the sensors to the femoral arterial catheters of the subjects. The arterial pressure output signal was incorporated into the Vigileo monitor for analysis of the waveform by the proprietary software of the manufacturer. Demographic data of each patient including age, gender, weight, and height were entered into the system. The sensor was zeroed and leveled at the same height as of right atrium for calibration. The arterial waveform fidelity was verified and thereafter measurements

Table 1. Patient characteristics.

\begin{tabular}{ll}
\hline Features & Number \\
\hline Number of patients (male/female) & $10(5 / 5)$ \\
Receiving lonotropic support & 2 \\
Septic shock & 6 \\
Cardiogenic shock & 2 \\
Patients with no shock & 2 \\
Arrhythmia & 0 \\
Age yrs (mean \pm SD) & $65 \pm 12$
\end{tabular}


were taken. The FTV system automatically measured SVV, SV, and CO every 20 seconds.

The stroke volume was subsequently measured using the portable Sonosite M-Turbo echocardiogram by a certified echocardiography technologist with more than 20 years of experience. Only 1 echo machine was used throughout the study. The transducer was 5-1 MHz P21x model. We used biplane Simpson's method of discs to calculate SV, in which subtraction of end-systolic volume from end-diastolic volume yields the SV (Fig. 3). Several acoustic window images were obtained and reviewed. The values of the best acoustic windows were averaged by the echo technologist and the supervising intensivist. On average, 2 measurements of the windows that had the highest contrast and resolution were made and averaged. The measurements were done at end expiration. Cardiac output was calculated by multiplying stroke volume by heart rate measured simultaneously at the time the stroke volume was being measured. No patient had any arrhythmia during the measurements. The echocardiographic measures were obtained between 6 and 48 hours after FTV system was placed. The echo and FTV measurements for each subject were done simultaneously.

The Simpson's methodology was used as follows (Fig. 3): (1) We obtained an apical 4 chamber view. (2) Then we traced the left ventricular cavity at ventricular end-diastole (end-diastole can be defined as the onset of the QRS complex, the frame after mitral valve closure or the frame in the cycle in which the cardiac chamber dimension is largest). (3) We then measured the left ventricular end-diastolic length from the mid mitral annulus to the cardiac apex. This yielded the left ventricular end-diastolic volume in mL. (4) We repeated the same steps at end-systole (end-systole is defined as the frame preceding mitral valve opening or the time in the cardiac cycle in which the cardiac chamber dimension is smallest to determine the end-systolic volume. (5) We then acquired apical 2-chamber view and the same end-diastolic and end-systolic measurements were performed. The calculation of end-diastolic volume minus end-systolic volume yields stroke volume. ${ }^{4,5}$

\section{Data collection and statistical analysis}

Data obtained by echocardiography, and FTV measurements were recorded and analyzed offsite by one investigator blinded to the study. Descriptive statis- tics (means and standard deviations) were computed with comparisons made between techniques for cardiac output and stroke volume values using Student $t$ tests $($ alpha $=0.05)$. In addition, Pearson correlation coefficients were calculated to determine linearity between systems, and Bland-Altman plots were constructed to assess agreement between methods.

\section{Results}

Table 1 is the summary of patients' demographics and characteristics. Most of the patients had shock, and predominantly it was due to sepsis. These patients needed a clearer evaluation of their volume status as it was unclear if patients were hypovolemic, euvolemic, or hypervolemic and if more ionotropic infusion, volume infusion, or both were needed. Therefore, an arterial catheter was inserted for FTV measurements, and values were obtained for SV and CO.

Table 2 shows the cardiac output and stroke volume comparisons between echocardiography and FTV methods. No significant differences were noted between the FTV and Sonosite methods for assessing $\mathrm{CO}$ or $\mathrm{SV}(P>0.05)$. The values of $\mathrm{CO}$ and $\mathrm{SV}$ measured by FTV and the echocardiogram correlated strongly $\left(R^{2}=0.98\right.$ and 0.97 , respectfully).

Figure 1 displays Bland-Altman plots for $\mathrm{CO}$ and SV as measured by echocardiography and FTV and demonstrates strong agreement between methods with no apparent bias.

Figure 2A depicts the correlation between stroke volume measurements with FTV and Sonosite echocardiogram. The $R^{2}$ value of 0.98 shows a robust correlation. (For SV, $\mathrm{y}=0.9545 \mathrm{x}+3.3, \mathrm{R}^{2}=0.98$ and for $\left.\mathrm{CO}, \mathrm{y}=0.9104 \mathrm{x}+7.7074, \mathrm{R}^{2}=0.97\right)$.

Figure 2B demonstrates the cardiac output differences between FTV and Sonosite echocardiogram with an $R^{2}$ value of 0.97 , again indicating a strong correlation.

\section{Discussion}

Our data show the following: (1) there is good correlation between the FTV and portable bedside echocar-

Table 2. Cardiac output and stroke volume measures $($ mean $\pm \mathrm{SD})$.

\begin{tabular}{llll}
\hline & Echo & Vigileo & $P$-value \\
\hline CO $(\mathrm{L} / \mathrm{m})$ & $4.99 \pm 0.71$ & $4.98 \pm 0.62$ & 0.97 \\
SV $(\mathrm{mL})$ & $85.5 \pm 24.3$ & $84.8 \pm 25.9$ & 0.95 \\
\hline
\end{tabular}




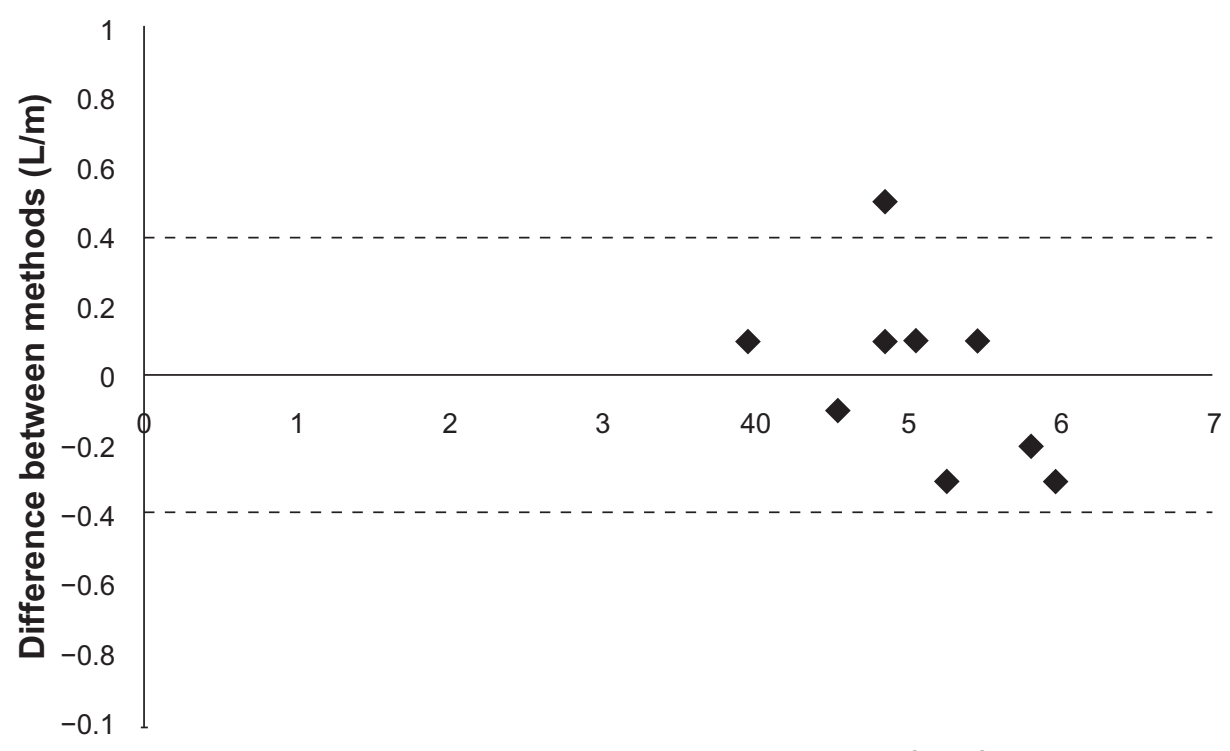

Average mean cardiac output (L/m)

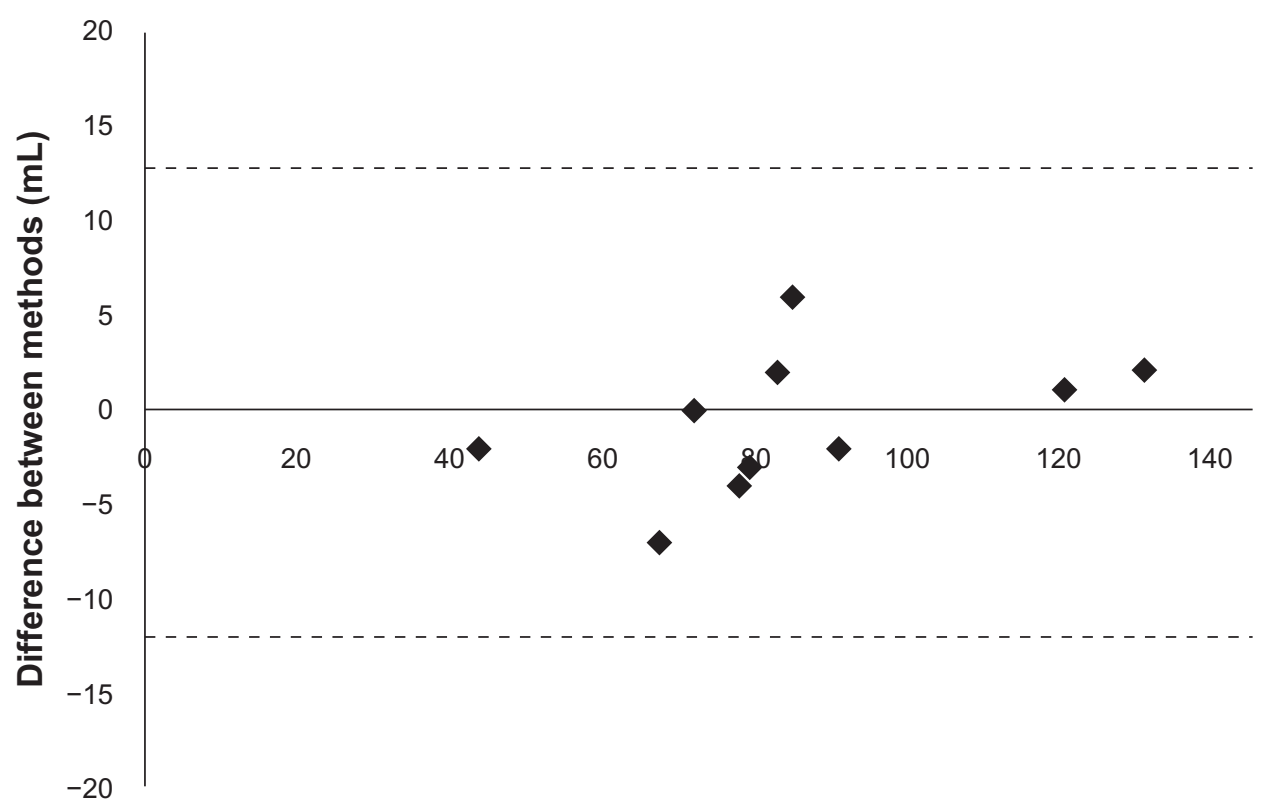

Average mean stroke volume $(\mathrm{mL})$

Figure 1. The variation between cardiac output and stroke volume measured by FTV and Sonosite transthoracic echocardiogram. The $95 \%$ limits of agreement (2SD) are shown by the broken lines.

diography in measurement of stroke volume and cardiac output in patients who are on mechanical ventilation and (2) portable bedside echocardiography can be reliable and helpful in noninvasive measurements of preload and thus help guide clinical decision making.

To our knowledge, there is no published study that compares FTV with SV measurements to the Sonosite potable echocardiogram. FTV has been compared with conventional echocardiography in previous studies. McLean et $\mathrm{al}^{6}$ demonstrated that there was poor comparability between echo-derived and FTV-derived cardiac output in their patients. However, after exclusion of atrial fibrillation and aortic stenosis, the comparability became more favorable between the two groups with a bias and percentage error that was within the $30 \%$ limit of clinical acceptability as suggested by a previous study. ${ }^{?}$

Biais et al showed SVV between FTV and echocardiography in liver transplant patients. Their study showed an acceptable bias and limits of agree- 
A
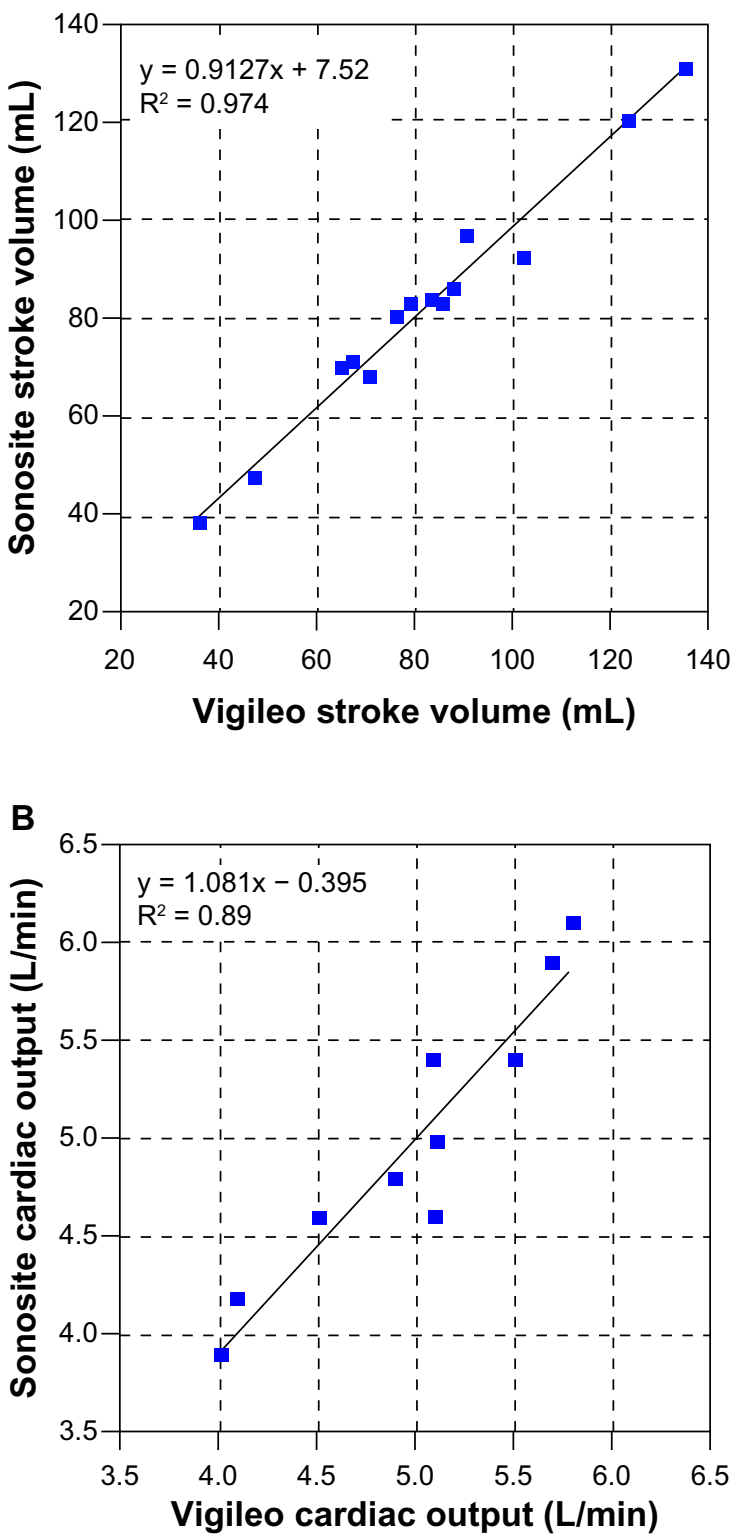

Figure 2. (A) Correlation of stroke volume between FTV and Sonosite transthoracic echocardiogram. (B) Correlation of Cardiac output between FTV and Sonosite transthoracic echocardiogram.

ment and similar performance when it came to fluid responsiveness. ${ }^{8}$ FTV has also been compared with other methods for cardiac output measurement such as thermodilution, which is considered to be gold standard in cardiac output measurements. The correlation was good and the results were comparable. ${ }^{9}$ Other studies have shown validation of FTV to thermodilution and another arterial pulse contour-based cardiac output. ${ }^{10}$ Some consider the Fick rather than the thermodilution method to be the gold standard in cardiac output measurement. However, a controlled head-to-head study comparing Fick with FTV is not available as of yet. ${ }^{11}$

Echocardiography is an important tool in assessment of the hemodynamic state of patients in intensive care units. ${ }^{12}$ It is noninvasive, well tolerated, and readily available. Basic critical care echocardiography is now being recognized as an important technique that is being emphasized as an essential learning tool for critical care physicians and not just in the domain of cardiologists. ${ }^{13}$

Limitations of conventional echocardiography systems include their size and lack of training for utilization by the traditional intensivists. In an optimal scenario, an ordered echocardiographic study is done by a certified echo technician and read by a cardiologist. This approach has significantly dissuaded critical care physicians from ordering and utilizing this considerably important tool in intensive care units. The lack of fundamental critical care echocardiography knowledge by most of intensivists has also limited the more extensive use of this quickly evolving method. In some countries in Europe, an echo machine is not only utilized by cardiologists but also by intensivists, who are better trained than some of their counterparts in North America. ${ }^{14}$

It appears that a portable echocardiogram such as the one manufactured by Sonosite and used in this study will be an attractive alternative to the traditional large echo machines. The Sonosite model has several advantages: it is light, portable, and technologically not complicated like the traditional larger echo machines. The above features make the equipment very attractive for mastery by pulmonologists and critical care physicians with an interest in bedside assessment of hemodynamics.

The views that are important in TTE (Transthoracic echocardiography) for a critical care physician are an apical 4-chamber view to detect right ventricular dilatation, and, with pulsed Doppler, it will record left ventricular inflow for assessment of left ventricular filling pressure. ${ }^{14}$ Another view is the parasternal short axis view used for evaluation of LV contractility and for calculation of left ventricle fractional area contraction. ${ }^{14}$ In addition, a subcostal view can assess the inferior vena cava (IVC). A small IVC is highly suggestive of hypovolemia and the need for volume replenishment, while a dilated or congestive IVC is suggestive of heart failure and fluid overload. ${ }^{14}$

Based on a literature search, Sonosite echocardiogram has not been compared with FTV 

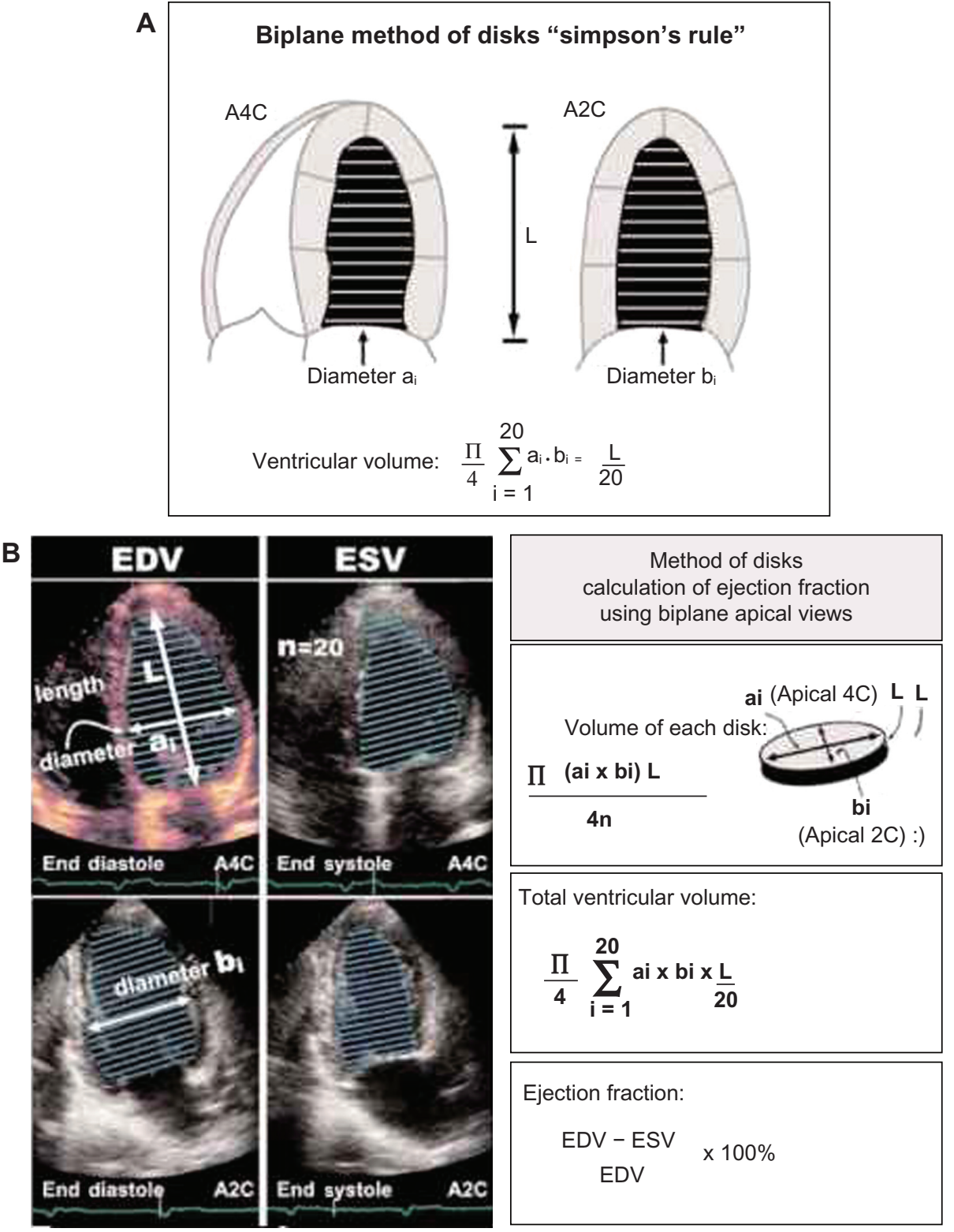

Figure 3. The Simpson's method for measurement of cardiac output. http://www.barnardhealth.info/echocardiography/a4c.html.

monitoring previously. Our study shows a strong correlation between the two systems for assessment of stroke volume with a percentage error that was reasonably acceptable. This was also true for cardiac output measurement. We used the Simpson's method of discs for stroke volume measurement unlike a previous study that utilized the left volume outflow tract (LVOT) method with flow used for measurement of cardiac output. ${ }^{6}$ The FTV data validate the data obtained from Sonosite. The LVOT has limitations in calculating stroke volume in patients with LVOT dynamic obstruction or patients with significant aortic regurgitation. Calculating stroke volume with LVOT in such patients is not valid. None of our patients had such findings. Otherwise, both methods are considered to be valid in measurement of stroke volume.

Limitations of our study are as follows: (1) the size of our group is small, and a larger number of subjects is needed; (2) the patients are mechanically ventilated, and validation of data in nonmechanically ventilated patients is also needed, which is a limitation because FTV is only validated in patients on mechanical ventilation and may not be reliable in patients who are not mechanically ventilated; (3) the measurements with echocardiography were done only once, and multiple measurements at different times and on different days 
were not made. It would have been ideal if several echocardiographic measurements at different intervals were being made and all of those were compared with the FTV system.

\section{Acknowledgements}

The abstract of this paper was previously published as a meeting abstract in Chest 2012;142.

\section{Author Contributions}

Conceived and designed the experiments: MB, LF, SP. Analyzed the data: MB, MB, HT. Wrote the first draft of the manuscript: MB. Contributed to the writing of the manuscript: MB, MB, SP. Agree with manuscript results and conclusions: MB. Jointly developed the structure and arguments for the paper: MB, LF. Made critical revisions and approved final version: MB. All authors reviewed and approved of the final manuscript.

\section{Funding}

Author(s) disclose no funding sources.

\section{Competing Interests}

Author(s) disclose no potential conflicts of interest.

\section{Disclosures and Ethics}

As a requirement of publication the authors have provided signed confirmation of their compliance with ethical and legal obligations including but not limited to compliance with ICMJE authorship and competing interests guidelines, that the article is neither under consideration for publication nor published elsewhere, of their compliance with legal and ethical guidelines concerning human and animal research participants (if applicable), and that permission has been obtained for reproduction of any copyrighted material. This article was subject to blind, independent, expert peer review. The reviewers reported no competing interests.

\section{References}

1. Chatterjee K. The Swan-Ganz catheters: past, present, and future. A viewpoint. Circulation. 2009;119(1):147-52.

2. Sander M, Spies CD, Grubitzsch H, Foer A, Muller M, von Heymann C. Comparison of uncalibrated arterial waveform analysis in cardiac surgery patients with thermodilution cardiac output measurements. Crit Care. 2006;10(6):R164.

3. Chew MS. Haemodynamic monitoring using echocardiography in the critically ill: a review. Cardiol Res Pract. 2012;2012:139537.

4. Huntsman LL, Stewart DK, Barnes SR, Franklin SB, Colocousis JS, Hessel EA. Noninvasive Doppler determination of cardiac output in man. Clinical validation. Circulation. 1983;67(3):593-602.

5. Otterstad JE, Froeland G, St. John Sutton M, Holme I. Accuracy and reproducibility of biplane two-dimensional echocardiographic measurements of left ventricular dimensions and function. Eur Heart J. 1997;18(3): 507-13.

6. McLean AS, Huang SJ, Kot M, Rajamani A, Hoyling L. Comparison of cardiac output measurements in critically ill patients: FloTrac/Vigileo vs. transthoracic Doppler echocardiography. Anaesth Intensive Care. 2011;39(4): 590-8.

7. Critchley LA, Critchley JA. A meta-analysis of studies using bias and precision statistics to compare cardiac output measurement techniques. J Clin Monit Comput. 1999;15(2):85-91.

8. Biais M, Nouette-Gaulain K, Roullet S, Quinart A, Revel P, Sztark F. Acomparison of stroke volume variation measured by Vigileo/FloTrac system and aortic Doppler echocardiography. Anesth Analg. 2009;109(2):466-9.

9. McGee WT, Horswell JL, Calderon J, et al. Validation of a continuous, arterial pressure-based cardiac output measurement: a multicenter, prospective clinical trial. Crit Care. 2007;11(5):R105.

10. de Waal EE, Kalkman CJ, Rex S, Buhre WF. Validation of a new arterial pulse contour-based cardiac output device. Crit Care Med. 2007;35(8):1904-9.

11. Pugsley J, Lerner AB. Cardiac output monitoring: is there a gold standard and how do the newer technologies compare? Semin Cardiothorac Vasc Anesth. 2010;14(4):274-82.

12. Ozier Y, Gueret P, Jardin F, Farcot JC, Bourdarias JP, Margairaz A. Two-dimensional echocardiographic demonstration of acute myocardial depression in septic shock. Crit Care Med. 1984;12(7):596-9.

13. Mayo PH. Training in critical care echocardiography. Ann Intensive Care. 2011;1:36.

14. Au SM, Vieillard-Baron A. Bedside echocardiography in critically ill patients: a true hemodynamic monitoring tool. J Clin Monit Comput. 2012;26(5):355-60. 